let $x$ be fixed, $-1<x<1$. We obtain for the roots of the polynomial (19) in $z$ the condition

$$
\frac{1+x z}{\left(1+2 x z+z^{2}\right)^{1 / 2}}=x_{\nu}
$$

where $x_{\nu}$ denotes a root of $P_{n}$. Or

$$
z=\frac{x\left(x_{\nu}^{2}-1\right) \pm x_{\nu}\left(\left(1-x_{v}^{2}\right)\left(1-x^{2}\right)\right)^{1 / 2}}{x^{2}-x_{\nu}^{2}}
$$

thus the roots in $z$ are all real. Using the trivial inequality (16) the assertion follows.

STANFORD UNIVERSITY

\title{
NOTE ON THE EIGENVALUES OF THE STURM-LIOUVILLE
} DIFFERENTIAL EQUATION

GERALD FREILICH

In discussing eigenvalues and eigenfunctions of the Sturm-Liouville differential equation

$$
L(u)+\lambda \rho u=0, \quad L(u)=\left(p u^{\prime}\right)^{\prime}-q u,
$$

with

$$
\left.\begin{array}{rl}
p(x) & \geqq m>0 \\
q(x) & \geqq 0 \\
\beta & \geqq \rho(x) \geqq \alpha>0
\end{array}\right\} \quad \text { for } a \leqq x \leqq b, \text { and for some } \alpha, \beta, \text { and } m,
$$

and the boundary conditions

$$
u(a)=c_{1} u(b), \quad u^{\prime}(a)=c_{2} u^{\prime}(b), \quad c_{1} c_{2} p(a)=p(b),
$$

we find that we can represent our eigenfunctions as unit normals in the directions of the principal axes of an ellipsoid in function space. We define our function space $F$ as the set of all functions $v(x)$, $a \leqq x \leqq b$, which satisfy the boundary conditions of the Sturm-Liouville equation. The origin of our space will be the function $u(x)=0$. We can now metrize $F$ by defining our inner product $(u, v)$ for

Received by the editors June 26, 1947. 
$u \in F, v \in F$, as

$$
(u, v)=\int_{a}^{b} \rho u v d x
$$

Also $|u|=((u, u))^{1 / 2}$, and $u$ is orthogonal to $v$ if and only if $(u, v)=0$. Let me now define

$$
D(u, v)=\int_{a}^{b}\left(p u^{\prime} v^{\prime}+q u v\right) d x .
$$

Notice $D(u, u)=\int_{a}^{b}\left(p\left(u^{\prime}\right)^{2}+q u^{2}\right) d x \geqq 0$.

In terms of $D, I$ shall define an infinite-dimensional ellipsoid in our function space. Take a unit vector in $F$, that is, $(u, u)=1$. On this, lay off a length $r=1 /(D(u, u))^{1 / 2}$. The set of points of $F$ thus determined for all unit vectors of $F$ constitutes the ellipsoid. It can be shown that the unit normals in the directions of the principal axes constitute a complete orthonormal set of eigenfunctions. Furthermore, if we arrange this set such that $u_{1}$ is the unit vector in the direction of the longest principal axis, $u_{2}$ the unit vector in the direction of the next longest principal axis, and so on, then $\lambda_{1}=D\left(u_{1}, u_{1}\right)$, $\lambda_{2}=D\left(u_{2}, u_{2}\right), \cdots$ constitute an increasing set of eigenvalues, $\lambda_{i}$ being the eigenvalue of $u_{i}$.

To show that $\lambda_{n} \approx n^{2}$ as $n \rightarrow \infty$, we must define the $n$th principal axis $u_{n}(x)$ independently of the previous $n-1$ principal axes $u_{i}(x)$, $i=1,2, \cdots, n-1$. This is customarily done in the following manner:

Take a set of $n-1$ linearly independent vectors of $F, v_{1}, v_{2}, \cdots$, $v_{n-1}$, through the origin and consider all unit vectors $u \perp v_{i}$, $i=1,2,3, \cdots, n-1$. Hence $(u, u)=1,\left(u, v_{i}\right)=0$. Let $\min D(u, u)$ $=f\left(v_{i}\right)$. Then $\max f\left(v_{i}\right)=\lambda_{n}$.

The way I define $\lambda_{n}$ runs as follows:

Let $v_{1}, v_{2}, \cdots, v_{n}$ be a set of $n$ linearly independent vectors of $F$. Consider

$$
u=c_{1} v_{1}+c_{2} v_{2}+\cdots+c_{n} v_{n} .
$$

Then we normalize $u$ getting $u /|u|$. It is easy to see that $D(u /|u|$, $u /|u|)=D(u, u) /(u, u)$. Now let $g\left(v_{i}\right)=\max D(u, u) /(u, u)$ for all $u$ as defined above. Then

$$
\min g\left(v_{i}\right)=\lambda_{n}
$$

To prove this, let $v_{i}=u_{i}$, for $i=1,2, \cdots, n$. Then

$$
(u, u)=\left(\sum c_{i} u_{i}, \sum c_{j} u_{j}\right)=\sum c_{i} c_{j}\left(u_{i}, u_{j}\right)=\sum c_{i}^{2}
$$




$$
\frac{D(u, u)}{(u, u)}=\frac{D\left(\sum c_{i} u_{i}, \sum c_{j} u_{j}\right)}{\sum c_{i}^{2}}=\frac{\sum c_{i}^{2} \lambda_{i}}{\sum c_{i}^{2}} \leqq \frac{\sum c_{i}^{2} \lambda_{n}}{\sum c_{i}^{2}}=\lambda_{n},
$$

since $\lambda_{i} \leqq \lambda_{n}$ for $i=1,2, \cdots, n-1$. Hence $g\left(u_{i}\right) \leqq \lambda_{n}$. However, if $c_{i}=0$, for $i=1,2, \cdots, n-1, c_{n}=1$, then $u=u_{n}$, and hence $D(u, u) /(u, u)=\lambda_{n}, g\left(u_{i}\right)=\lambda_{n}$. To complete the proof I must show that for all $v_{i}, g\left(v_{i}\right) \geqq \lambda_{n}$.

Let $u=c_{1} v_{1}+c_{2} v_{2}+\cdots+c_{n} v_{n}$ be such that $u \perp u_{i}$ for $i=1, \cdots$, $n-1$, that is, $\left(u, u_{i}\right)=0=c_{1}\left(u_{i}, v_{1}\right)+\cdots+c_{n}\left(u_{i} v_{n}\right)$. This is always possible since I have $n-1$ equations in $n$ unknowns. Now by the definition of $u_{n}$, for all vectors $u \perp u_{i}, i=1,2, \cdots, n-1$,

$$
\lambda_{n}=D\left(u_{n}, u_{n}\right) \leqq \frac{D(u, u)}{(u, u)} .
$$

Hence $g\left(v_{i}\right)=\max D(u, u) /(u, u) \geqq \lambda_{n}$.

With this definition, we can now develop inequalities for the eigenvalues. Consider

$$
\begin{aligned}
& \left(p_{1} u^{\prime}\right)^{\prime}-q_{1} u+\lambda \rho_{1} u=0 \\
& \left(p_{2} u^{\prime}\right)^{\prime}-q_{2} u+\mu \rho_{2} u=0
\end{aligned}
$$

with

$$
p_{1}(x) \leqq p_{2}(x), \quad q_{1}(x) \leqq q_{2}(x), \quad \rho_{1}(x) \geqq \rho_{2}(x) \quad \text { for } a \leqq x \leqq b,
$$

and with both equations satisfying similar boundary conditions. Denoting the eigenvalues of (1) by

and those of (2) by

$$
\lambda_{1}, \lambda_{2}, \lambda_{3}, \cdots
$$

$$
\mu_{1}, \mu_{2}, \mu_{3}, \cdots,
$$

we shall prove that $\lambda_{n} \leqq \mu_{n}$ for $n=1,2, \cdots$.

Let $v_{1}, v_{2}, \cdots, v_{n}$ be a fixed set of $n$ independent vectors. Let $u=c_{1} v_{1}+\cdots+c_{n} v_{n}$. For (1) the normalized $u$ is $u /|u|_{1}$ and $D_{1}\left(u /|u|_{1}, u /|u|_{1}\right)=D_{1}(u, u) /(u, u)_{1}, g_{1}\left(v_{i}\right)=\max D_{1}(u, u) /(u, u)_{1}$. For (2) the normalized $u$ is $u /|u|_{2}$, and $g_{2}\left(v_{i}\right)=\max D_{2}(u, u) /(u, u)_{2}$. Since $D_{1}(u, u)=\int\left(p_{1}\left(u^{\prime}\right)^{2}+q_{1} u^{2}\right) d x, D_{2}(u, u)=\int\left(p_{2}\left(u^{\prime}\right)^{2}+q_{2} u^{2}\right) d x$, it follows that $D_{2}(u, u) \geqq D_{1}(u, u)$. Since $(u, u)_{1}=\int \rho_{1} u^{2} d x,(u, u)_{2}$ $=\int \rho_{2} u^{2} d x$, it follows that $(u, u)_{1} \geqq(u, u)_{2}$. Hence $D_{2}(u, u) /(u, u)_{2}$ $\geqq D_{1}(u, u) /(u, u)_{1}, g_{2}\left(v_{i}\right) \geqq g_{1}\left(v_{i}\right)$. Since min $g_{2}\left(v_{i}\right)=\mu_{n}$, and $\min g_{1}\left(v_{i}\right)$ $\lambda_{n}$, then $\mu_{n} \geqq \lambda_{n}$.

With these preliminaries, the proof about the asymptotic behavior of $\lambda_{n}$ is standard. 
An example of the use of this new definition of $\lambda_{n}$ is that it affords a method of obtaining upper bounds and approximations to $\lambda_{n}$.

Take $v_{i}=v_{i}\left(x, a_{1}, a_{2}, \cdots, a_{r}\right)$, with $r$ parameters, such that $v_{i}$ for $i=1,2, \cdots, n$ satisfies the boundary conditions, that is, it is a vector in function space. Then form

$$
u=c_{1} v_{1}+\cdots+c_{n} v_{n},
$$

and normalize $u$, getting

$$
(u, u)=\sum c_{i} c_{j}\left(v_{i}, v_{j}\right)=1 .
$$

We can now calculate $\max D(u, u)$, for

$$
D(u, u)=\sum c_{i} c_{j} D\left(v_{i}, v_{j}\right),
$$

and hence we get

$$
\frac{\partial\left[\sum c_{i} c_{j} D\left(v_{i}, v_{j}\right)\right]}{\partial c_{i}}=\sigma \frac{\partial\left[\sum c_{i} c_{j}\left(v_{i}, v_{j}\right)\right]}{\partial c_{i}},
$$

which together with the equation

$$
\sum c_{i} c_{j}\left(v_{i}, v_{j}\right)=1
$$

determine the $c_{i}$.

Hence $\max D(u, u)=h\left(a_{1}, a_{2}, \cdots, a_{r}\right) \geqq \lambda_{n}$. Hence to approximate $\lambda_{n}$, we minimize the function $h\left(a_{1}, a_{2}, \cdots, a_{r}\right)$. Good approximations depend of course on the choice of the original $v_{i}$. It is to be noticed that this is just an extension of the ordinary method of computing $\lambda_{0}$.

BROWN UNIVERSITY 